\title{
COMPARISON AND CALCULATION OF DIFFERENT ATMOSP- HERIC DELAY MODELS USING ATMOSPHERIC PARAMETERS OF VLBI STATIONS OF CHINA
}

\author{
ZHIGEN YANG \\ Shanghai Observatory, Academia Sinica \\ Shanghai 200030 \\ China
}

ABSTRACT. The values of the atmospheric time delay for the "Chao", "Marini" and "CfA-2.2" mapping function are calculated by using the atmospheric parameters in summer and winter at Shanghai, Kunming and Urumqi station respectively. A comparison among these values shows that the derivations of "Marini" from "Chao" and " $C f A$ " are relatively large. On the other hand, the difference of values between the "Chao" and the "CfA" in the case of $\epsilon=40^{\circ} \sim 10^{\circ}$, which is the average for the three stations, is from $+1 \mathrm{~mm}$ to $+47 \mathrm{~mm}$ for the "wet" part of the delay in summer, while is from $-2 \mathrm{~mm}$ to $-28 \mathrm{~mm}$ for the "dry" part in winter. For the case of low elevation angle $\epsilon \doteq 5^{\circ}$, the difference for the "wet" part can be about 400 $\mathrm{mm}$ in summer. Therefore, it is indispensable to make a further comparison between "Chao" and "CfA" mapping function by using the data of VLBI observations, in order to make a better revision to the adopted models of atmospheric delay.

The monthly averages of the height of tropopause $\bar{h}_{t}$ and the tropospheric temperature lapse rate $\bar{\beta}_{1}$ for the three stations mentioned above are used to calculate the dry atmospheric delay by the "CfA" mapping function. The results show that the amplitudes of the annual changing of delay $\mathrm{d} \tau_{a}$, which is caused by $\bar{h}_{t}$ and $\bar{\beta}_{t}$ for the case of $\epsilon=20^{\circ} \sim 10^{\circ}$ at Urumqi station, are about $1 \sim 5 \mathrm{~mm}$ and $2 \sim 15$ $\mathrm{mm}$ respectively. Therefore, taking the parameters of $\bar{h}_{1}$ and $\bar{\beta}_{1}$ of the stations into account in "CfA" model, instead of using fixed constants, would be much favourable for the requirements of 1 ps precision of VLBI physical models. 